\title{
Measurement of the magneto-optical correlation length in turbid media
}

\author{
Ralf Lenke, Christoph Eisenmann, Daniel Reinke, and Georg Maret \\ Department of Physics, University of Konstanz, Box 5560, 78457 Konstanz, Germany
}

(Received 21 May 2002; published 22 November 2002)

\begin{abstract}
In multiple light scattering media, magnetic field induced circular birefringence (Faraday effect) influences interference effects such as speckle pattern or coherent backscattering. It was predicted that in the diffusive regime the relevant correlation length with respect to the Faraday rotation $\ell_{F}^{\star}$ differs, in general, from the transport mean free path $\ell^{\star}$. We have experimentally verified the prediction that the ratio $\ell_{F}^{\star} / \ell^{\star}$ equals 2 for Rayleigh scattering and decreases to 1 with increasing scatterer size. We also discuss the influence of the structure factor on $\ell_{F}^{\star}$.
\end{abstract}

DOI: 10.1103/PhysRevE.66.056610

PACS number(s): 42.25.Dd, 78.20.Ls

\section{INTRODUCTION}

Magnetic field effects have significantly improved the understanding of electron transport in semiconductors and metals. For example, the observation of a magnetic field induced increase of the conductivity can be explained by a field induced destruction of weak localization. For a detailed description of this phenomenon which is based on the timereversal symmetry of (quantum) wave paths the reader is referred to [1] and references there.

In the case of electromagnetic wave transport numerous magnetic field effects [2-5] have been discovered in recent years, in particular in the visible range: magnetic field dependent speckle correlation [3], destruction of the coherent backscattering (CB) cone, the so-called precursor of weak localization [2,6,7], and the magneto-optical Hall effect [8]. The observed destruction of weak localization of light suggests the relevance of magneto-optical effects in the investigations of strong localization of electromagnetic waves [9-14].

In some of those experiments $[3,6]$ the magnetic field effect was stronger than initially [2] expected. Therefore it was suggested $[6,15]$ that the correlation length of the magnetic field induced phase shift differs from the transport mean free path $\ell^{\star}$. In this work, we experimentally determine the "new" characteristic length $\ell_{F}^{\star}$. This is done in a quantitative way for different size parameters of polystyrene spheres in water. Finally, we discuss the effect of the structure factor on $\ell_{F}^{\star}$ in the case of large volume fractions.

In order to fully understand the magnetic field effects mentioned above, one needs a quantitative comprehension of how Faraday rotation operates on the length scale $\ell^{\star}$. To this end, a very precise understanding of coherent backscattering in connection with the magnetic field effects is of general importance.

We have studied the length $\ell_{F}^{\star}$ by exploiting CB not only because $\mathrm{CB}$ is the precursor to weak localization but also because it provides the most precise determination of $\ell_{F}^{\star}$. Nevertheless, the considerations are also valid for magnetic field dependent speckle correlation measurements.

\section{THEORY}

CB originates from the constructive interference between each light path and its reversed path. In the exact back- scattering direction $\left(\vec{k}_{f}=-\vec{k}_{i}\right.$ for final and incident wave vectors) both paths always have exactly the same length, thus leading to a constructive interference enhancement of a factor of 2 in the exact backscattering direction with respect to the "incoherent background." For more details, see Ref. [16], for example. Out of exact backscattering a phase shift of $\left(\vec{k}_{f}+\vec{k}_{i}\right) \cdot \vec{\rho}$ is introduced, where $\vec{\rho}$ is the distance vector between the start and end points of a light path on the surface of the sample. This phase shift results in a decrease of the CB enhancement, which decays to the incoherent background at wide angles on average over all light paths. For simplicity, we neglect the vector character of the light as well as single scattering here. The shape of the so-called CB cone $E\left(\overrightarrow{q_{b}}\right)$ as a function of the "backscattering vector" $\vec{q}_{b}:=\vec{k}_{f}$ $+\vec{k}_{i}$ is given by the Fourier transform of the intensity distribution $I(\vec{\rho})[17,18]$ :

$$
E\left(\vec{q}_{b}\right)=1+\int I(\vec{\rho}) \cos \left(\vec{q}_{b} \cdot \vec{\rho}\right) d \vec{\rho}
$$

In the diffusion approximation one obtains [17]

$$
E\left(q_{b}\right) \approx 1+\int p(s) \exp \left(-\frac{1}{3} s \ell^{\star} q_{b}^{2}\right) d s,
$$

where $p(s)$ is the path length distribution. Faraday rotation (FR) introduces a phase shift of $\pm V \vec{B} \cdot \vec{r}$ for circularly polarized light (handedness \pm 1 ) propagating a distance $\vec{r}$ parallel to a magnetic field $\vec{B} . V$ is the specific Verdet constant. In the first models [2] describing the influence of FR on $\mathrm{CB}$, the average distance between two scattering events $\langle|\vec{r}|\rangle$ was approximated by $\ell^{\star}$ and it was assumed that the handedness changes randomly after each scattering event. Then the phase of a light amplitude experiences a mean square deviation of $\frac{1}{3} s \ell^{\star}(V B)^{2}$ along a path of length $s$. For small arguments, this phase shift can be included into Eq. (2) by an additional approximate factor $\exp \left[-\frac{1}{3} s \ell^{\star}(2 V B)^{2}\right]$. For a detailed description, see [6]. The factor 2 in front of $V B$ results from the fact that in the case of CB the phase shifts of counterpropagating waves add up. Thus, in the diffusion limit, due to the replacement of $q_{b}$ by $2 V B$ in the exponents in Eq. (2), the 


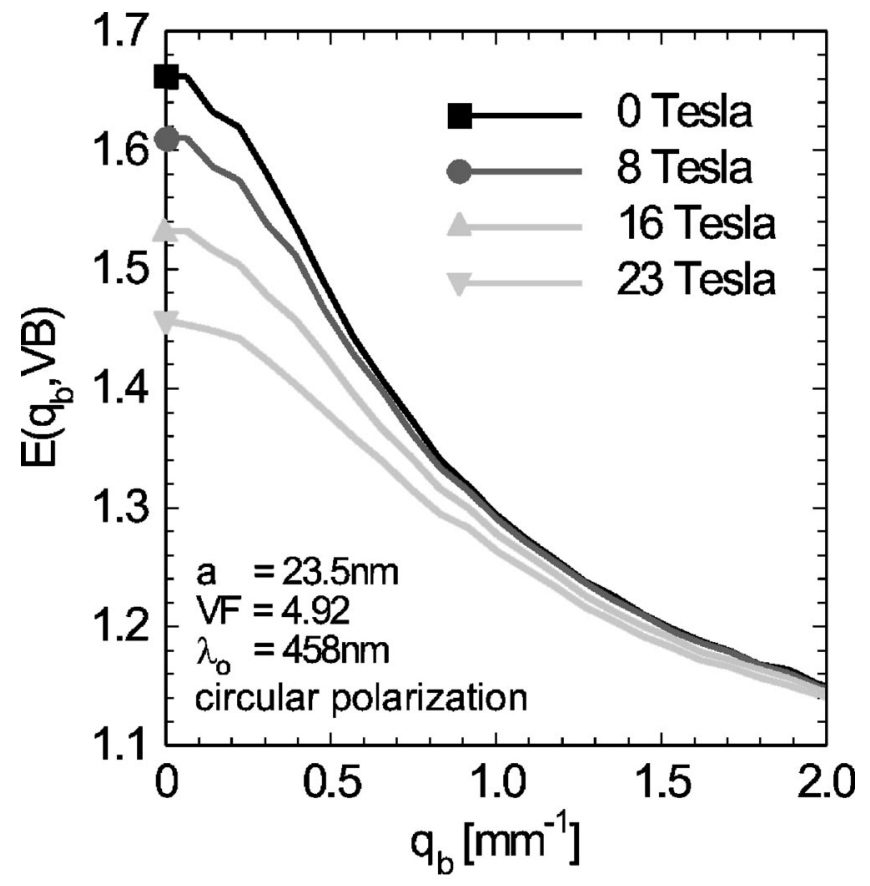

FIG. 1. (Color online only) Destruction of the CB cone with increasing magnetic field for a sample of polystyrene spheres (radius $a=23.5 \mathrm{~nm}, \mathrm{VF} 4.92 \%$ ) in water.

cone shape as a function of $q_{b}$ and $V B$ is obtained by the replacement $q_{b}^{2} \rightarrow q_{b}^{2}+q_{F}^{2}$, with $q_{F}^{2}:=(2 V B)^{2}$.

However, the approximation of a random helicity flip after each scattering event neglects correlations between depolarization and scattering angle. In fact, the circular depolarization is usually strongly correlated with the scattering angle $\theta$. In the case of Rayleigh scattering, for example, there is a complete helicity flip only for $\theta=\pi$. Therefore, it was suggested [6] to introduce a different correlation length for the influence of FR according to a modified differential cross section $d \sigma_{F}(\theta)=d \sigma^{++}(\theta)+d \sigma^{ \pm}(\theta-\pi)$, corresponding to the parts of the light that are scattered in the same ++ and in the orthogonal \pm circular polarization states, respectively. Therefore the correlation length is obtained in analogy to the definition of $\ell^{\star}$ in the case of nonisotropic scattering: $\ell_{F}^{\star} / \ell=(1-\langle\cos \theta\rangle)^{-1}$, where the average is taken according to $d \sigma_{F}$ now. Thus, for small arguments, in the diffusion approximation, the influence of FR on $\mathrm{CB}$ is given by the replacement $q_{b}^{2} \rightarrow q_{b}^{2}+\left(\ell_{F}^{\star} / \ell^{\star}\right) q_{F}^{2}$. Experimentally, the specific value $\ell_{F}^{\star} / \ell^{\star}$ can be obtained by measuring the $\mathrm{CB}$ cone without FR, $E\left(q_{b}\right)$, and comparing it to the maximum enhancement factors $E(V B)$ at $q_{b}=0$, as a function of $V B$. The rescaling factor $\ell_{F}^{\star} / \ell^{\star}$ is obtained independently of $p(s)$ and $\ell^{\star}$.

\section{EXPERIMENTS}

The basic experimental setup has already been described elsewhere [6,19]. Here, we used a 23 T magnet with a vertical bore. The sample was placed at a depth of about $400 \mathrm{~mm}$ in the center of the field and illuminated from the top using a $90^{\circ}$ deviating mirror. The rest of the CB setup (semitranspar-

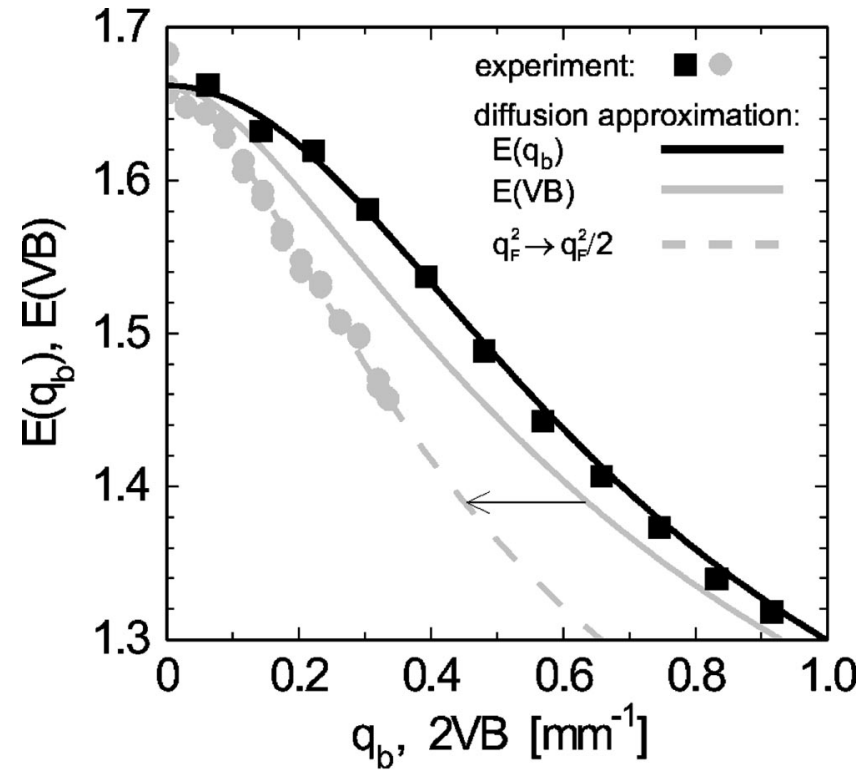

FIG. 2. (Color online only) Decrease of CB enhancement as a function of $q_{b}$ and $2 V B$. Marks, experiments; lines, theory. Same sample as in Fig. 1. The solid black line was fitted to the measured $E\left(q_{b}\right)$ (see text, fit parameters $d q_{\text {res }}=0.24 \mathrm{~mm}^{-1}, \gamma=2.3$ ). The same parameters have been used to calculate $E(2 V B)$ (solid gray line). However, as $\ell_{F}^{\star}>\ell^{\star}$, the measured curve $E(2 V B)$ decreases faster than expected. Rescaling in the argument (dashed line) gives the value $\ell_{F}^{\star} / \ell^{\star}=2$ here. Note that in case of a "perfect" experimental setup the solid gray and black lines should be identical.

ent mirror, beam expander, polarizers, lens of focal length $1330 \mathrm{~mm}$ in front of a charge-coupled device camera, $\mathrm{Ar}^{+}$ laser at $\lambda=458 \mathrm{~nm}$ ) was placed at a distance of about $2 \mathrm{~m}$ from this mirror, where magnetic field effects on the components of the setup could be neglected. The stability of the setup was verified by measuring the cone of a white Teflon sample positioned at the end of the magnet bore. For a precise angular calibration we measured the backscattering profile of a well defined glass sphere (diameter $5 \mathrm{~mm}$ ) [20,21]. The cell (diameter $40 \mathrm{~mm}$, height $28 \mathrm{~mm}$ ) containing the samples of polystyrene spheres suspended in water was open on top in order to avoid undesired effects (e.g., FR in glass windows, reflections).

In most samples the volume content of polystyrene beads was sufficiently small that the Faraday effect of pure water $\left(0.379^{\circ} / \mathrm{mm} \mathrm{T}\right.$ at $\left.\lambda=458 \mathrm{~nm}\right)$ could be used. At higher volume fractions we calculated the volume averaged Verdet constant $\left(V_{P S}=1.093^{\circ} / \mathrm{mm} \mathrm{T}\right.$ at $458 \mathrm{~nm}$ [22]). In order to obtain a significant magnetic field effect on the $\mathrm{CB}$ cone, the volume fraction (VF) of the polystyrene spheres was adjusted such that all samples had a transport mean free path of $\ell^{\star} \approx 500 \mu \mathrm{m}$. As an example, Fig. 1 shows the destruction of a $\mathrm{CB}$ cone with increasing magnetic field.

\section{EVALUATION OF EXPERIMENTAL RESULTS}

Due to the extreme experimental conditions (e.g., limited angular resolution of the setup, limited lateral intensity profile of the incident laser beam leading to slightly different 


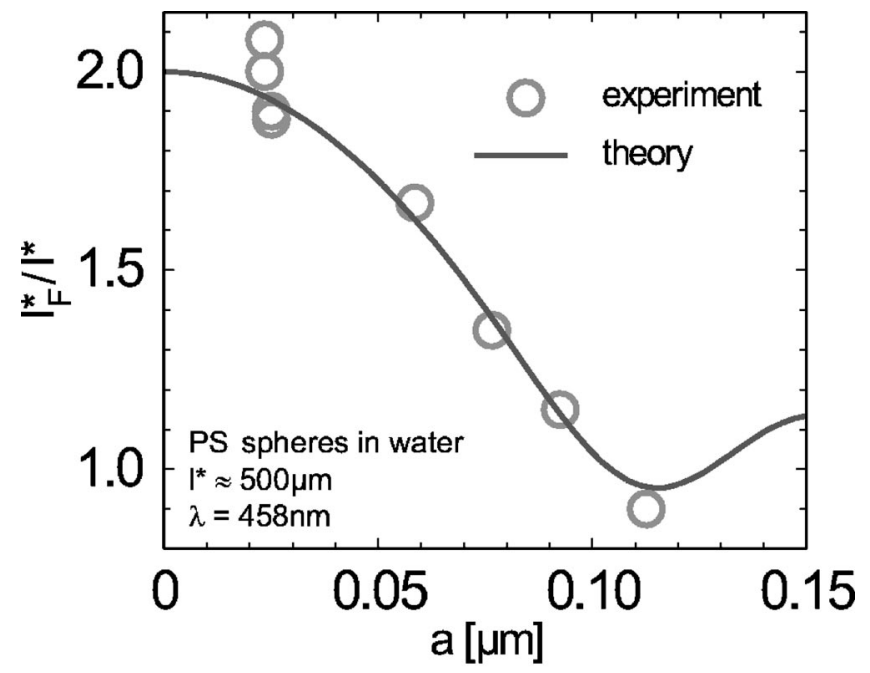

FIG. 3. (Color online only) Measured and calculated [6] values $\ell_{F}^{\star} / \ell^{\star}$ as a function of the polystyrene sphere radius $a$.

amplitudes in the direct and reversed paths) the measured cone shapes could not be compared directly to the analytical curves given by the earlier analysis in. Both experimental artifacts reduce the enhancement factor below 2 as can be seen in Fig. 1. The limited resolution $d q_{\text {res }}$ can be included approximately into Eq. (1) by a convolution in $\vec{q}_{b}$ with $\exp \left[-\left(\vec{q}_{b}^{\prime}-\vec{q}_{b}\right)^{2} / d q_{\text {res }}^{2}\right]$ (system response). The limited lateral intensity profile can be approximated by an additional convolution with $\exp \left[-\vec{R}^{2} / d R^{2}\right] \exp \left[-\left(\vec{R}^{\prime}-\vec{\rho}\right)^{2} / d R^{2}\right]$, where $\vec{R}$ and $\vec{R}^{\prime}-\vec{\rho}$ represent the start and end points of a random walk, and $d R$ reflects the width of the Gaussian intensity distribution (in our case $d R=6.1 \mathrm{~mm}$ ). Assuming a path length distribution $p(s)$ as suggested in $[17,18]$, integration over $\vec{q}_{b}$ and $\vec{R}$ can be performed analytically; the integration over $s$ was done numerically. The curve shape obtained was fitted to the experimental cone shape at zero field, $E\left(q_{b}\right)$, using $d q_{\text {res }}$ and $\gamma$ (see $\left.[17,18,25]\right)$ as free fit parameters. Then, the decrease of the cone tip $E(V B)$ as a function of the magnetic field was calculated using the same expression with the same parameters. Finally, the value $\ell_{F}^{\star} / \ell^{\star}$ was obtained by rescaling the calculated curve in the argument until best coincidence with the measured curve $E(V B)$ was found. Figure 2 shows the result for the same sample as in Fig. 1. We found that in our experiments $\ell_{F}^{\star} / \ell^{\star}$ is rather insensitive to the precise value of the fit parameters. The evaluation could be improved by simulating $p(s)$ in a scalar random walk model [19]. Figure 3 shows the result for different particle sizes. The coincidence with the theoretically predicted values of $\ell_{F}^{\star}$ is very good.

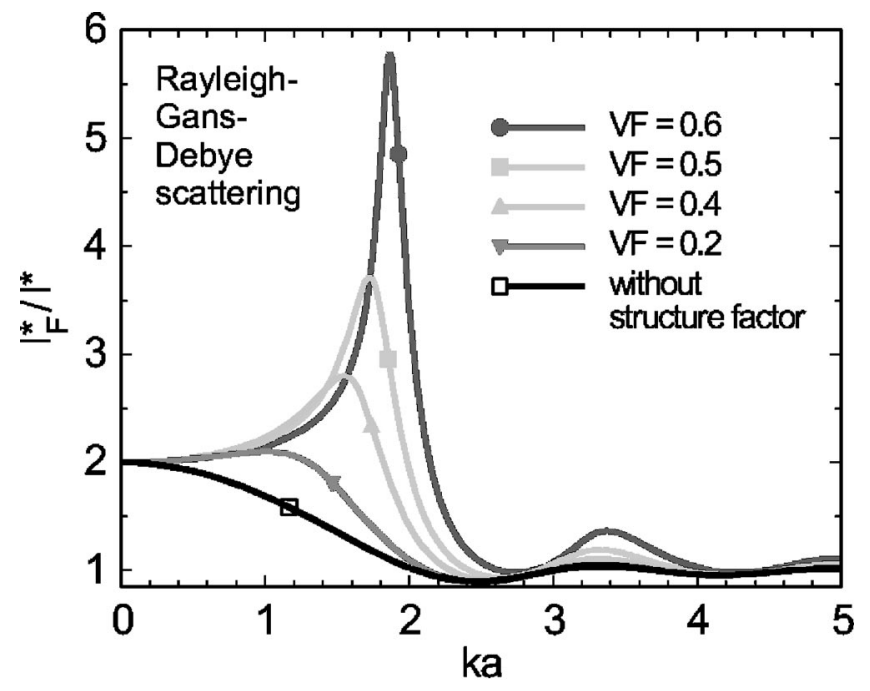

FIG. 4. (Color online only) $\ell_{F}^{\star} / \ell^{\star}$ as a function of size parameter for different volume fractions calculated for Rayleigh-GansDebye scattering including a Percus-Yevick structure factor.

\section{CONCLUSION AND OUTLOOK}

In conclusion, we have shown the importance of correlations between depolarization and scattering angle for multiple light scattering. They give rise to a strong scatterer size dependence of the relevant correlation length $\ell_{F}^{\star}$ divided by the transport mean free path $\ell^{\star}$. The suggested model [6] explains this dependence quite well and predicts the measured decrease of $\ell_{F}^{\star} / \ell^{\star}$ from 2 to about 1 in a quantitatively correct way.

Another interesting question concerns the role of positional correlations of scatterers as expressed by the structure factor. In our case, the samples with the smallest radius had a volume fraction of about $5 \%$, i.e., the structure factor may already be non-negligible (for the other samples the VF was always smaller than 1\%). In Fig. 4 we have calculated $\ell_{F}^{\star} / \ell^{\star}$ for Rayleigh-Gans-Debye scattering, including a PercusYevick structure factor $[23,24]$ in the differential scattering cross section. As can be seen, for $1{ }_{\sim}^{<} k a_{\sim}^{<} 2$ the value $\ell_{F}^{\star} / \ell^{\star}$ becomes larger than 2 and approaches nearly a value of 6 .

Consequently, $\ell_{F}^{\star}$ is a very useful parameter for studying light scattering in dense media by $\mathrm{CB}$ measurements or FR dependent speckle correlation measurements in transmission.

\section{ACKNOWLEDGMENTS}

The experiments were performed at the High Magnetic Field Laboratory, Grenoble. We thank Ralf Tweer, Thomas Gisler, and Anja Sparenberg for fruitful discussions, Stefanie Eiden who helped us in preparing the samples, and Geert Rikken who was our local contact at the GHMFL.
[1] G. Bergmann, Phys. Rev. 107, 1 (1984).

[2] A.A. Golubentsev, Sov. Phys. JETP 59, 26 (1984); F.C. MacKintosh and S. John, Phys. Rev. B 37, 1884 (1988).

[3] F.A. Erbacher, R. Lenke, and G. Maret, Europhys. Lett. 21,
551 (1993)

[4] A.S. Martinez and R. Maynard, Phys. Rev. B 50, 3714 (1994).

[5] B.A. van Tiggelen, Phys. Rev. Lett. 75, 422 (1995).

[6] R. Lenke and G. Maret, Eur. Phys. J. B 17, 171 (2000). 
[7] R. Lenke, R. Lehner, and G. Maret, Europhys. Lett. 52, 620 (2000)

[8] G.L.J.A. Rikken and B.A. van Tiggelen, Nature (London) 381, 54 (1996).

[9] D.S. Wiersma, P. Bartolini, A. Lagendijk, and R. Righini, Nature (London) 390, 671 (1997).

[10] F. Scheffold, R. Lenke, R. Tweer, and G. Maret, Nature (London) 398, 207 (1999).

[11] A.Z. Genack and N. Garcia, Phys. Rev. Lett. 66, 2064 (1991); A.A. Chabanov and A.Z. Genack, ibid. 87, 153901 (2001).

[12] R. Dalichaouch, J.P. Armstrong, S. Schultz, P.M. Platzman, and S.L. McCall Nature (London) 354, 53 (1991).

[13] R. Lenke, R. Tweer, and G. Maret, Eur. Phys. J. B 26, 235 (2002).

[14] P.W. Anderson, Philos. Mag. B 52, 505 (1985).

[15] D. Lacoste and B.A. van Tiggelen, Phys. Rev. E 61, 4556 (2000).

[16] R. Lenke and G. Maret, in Scattering in Polymeric and Colloidal Systems, edited by W. Brown and K. Mortensen (Gordon and Breach Scientific, New York, 2000), p. 1.

[17] P.E. Wolf, G. Maret, E. Akkermans, and R. Maynard, J. Phys. (France) 49, 63 (1988).

[18] E. Akkermans, P.E. Wolf, R. Maynard, and G. Maret, J. Phys. (France) 49, 77 (1988).

[19] C. Eisenmann, Diploma thesis, University of Konstanz, Germany 2002.

[20] R. Lenke, U. Mack, and G. Maret, J. Opt. A, Pure Appl. Opt. 4, 309 (2002).

[21] R. Lenke, R. Tweer, and G. Maret, J. Opt. A, Pure Appl. Opt. 4, 293 (2002).

[22] S. Muto, S. Ichikawa, N. Seki, and H. Ito, Electron. Commun. Jpn., Part 2: Electron. 74, 512 (1991).

[23] R. Klein, Interacting Brownian Particles: The Dynamics of Colloidal Suspensions (IOS Press, Amsterdam, 1997).

[24] S. Fraden and G. Maret, Phys. Rev. Lett. 65, 512 (1990).

[25] The factor $\gamma$ appears in the diffusion approximation of $p(s)$. Normally, its value is $\approx 0.7$. However, in the presence of internal reflections it may be larger. 